\title{
CONFÉRENCE
}

\section{Chirurgie pré-implantaire : les greffes autogènes} Dr Pierre Keller

Strasbourg

Une restauration implantaire esthétique et fonctionnelle implique une bonne gestion des tissus durs et des tissus mous. Compte tenu de sa capacité de cicatrisation et de sa prédictibilité, l'os autogène reste le " gold standard » pour la reconstruction de grands défauts alvéolaires.

Notre tecŸique de greffe est basée sur la préparation des blocs corticaux mandibulaires prélevés dans la zone rétromolaire. La forme de la nouvelle crête est reconstruite par de fines plaques corticales fixées à distance du site receveur. Le coffrage ainsi formé est comblé d'os particulaire, acteur principal du processus de régénération. Grâce à cette méthode, la transplantation de blocs mandibulaires constitue une méthode prévisible pour la régénération osseuse verticale. 